\title{
Investigation of a Radial Turbines Compatibility for Small Rocket Engine
}

\author{
Angelo Leto* \\ CIRA Italian Aerospace Research Center, via Maiorise, 81043 Capua (CE), Italy
}

Abstract. In the radial turbine preliminary design for an expander rocket engine, a comparison was made with axial turbine used in Pratt \& Whitney RL10 engine.

One of the primary requirements of a liquid propellant rocket engine is the generation of a high thrust, which depends on both the mass flow rate of the propellant and the pressure in the thrust chamber.

In expander-cycle engines, which are the subject of the present study, the liquid propellant is first compressed using centrifugal turbo-pumps, then it is used to cool the combustion chamber and the nozzle and, once vaporized, it flows through the turbines used to drive the turbo-pumps.

The aim was to demonstrate the greater efficiency of the radial turbine with a reduction of the pressure ratio with respect to the axial turbine.

\section{Nomenclature}

$\begin{array}{ll}\mathrm{c} & \text { Absolute Flow Velocity - Chord } \\ \mathrm{h} & \text { Enthalpy - Rotor Height } \\ \mathrm{L}_{\mathrm{ms}} & \text { Mean Surface Length } \\ \mathrm{M} & \text { Mach Number } \\ \mathrm{N}_{\mathrm{s}} & \text { Specific Speed } \\ \mathrm{O} & \text { Mean Throat Width } \\ \mathrm{p} & \text { Pressure } \\ \mathrm{q} & \text { Mean Blade Pitch }\end{array}$

$\begin{array}{ll}\mathrm{r} & \text { Radius } \\ \mathrm{T} & \text { Temperature } \\ \mathrm{u} & \text { Impeller Tangential Velocity } \\ \mathrm{w} & \text { Relative Velocity } \\ \mathrm{Y} & \text { Young's Modulus } \\ \mathrm{Z} & \text { Number Blades } \\ \mathrm{Z}_{\mathrm{r}} & \text { Rotor Axial Length }\end{array}$

Greek Letters

\begin{tabular}{llll}
$\alpha$ & Flow angle & $\sigma_{\mathrm{r}}$ & Rotor Blades Elastic Stress \\
$\beta$ & Relative Flow Angle & $\varphi$ & Flow Coefficient \\
$\eta$ & Efficiency & $\psi$ & Loading Coefficient \\
$v$ & Poisson's ratio & $\omega$ & Frequency \\
$\rho$ & Density & & \\
\multicolumn{2}{l}{ Subscripts } & & \\
0 & Total & $\mathrm{h}$ & hub \\
1 & Rotor Inlet & $\mathrm{s}$ & shroud \\
2 & Rotor Outlet & &
\end{tabular}

\section{Introduction}

The turbine design was carried out in two phases, implementing a meanline analysis model that uses an approximation one-dimensional for the flow through the machine and empirical correlations to estimate the losses, necessary for performance calculation.

The tool known as Radial Turbine Global Design RTGD [1] is used to obtain the rotor geometry main properties based on the performance required in RL10-3-3A [2] engine turbine design operating point. In addition, it calculates the main losses to determine efficiency useful for improving rotor design.

The tool can be used for radial turbines design for different applications, not just space. In this particular case, the working fluid is hydrogen, but it can be used for other fluids, since the thermodynamic properties are provided by the CoolProp libraries [3].

The model was validated using the available experimental data of RL10 [2] expander-cycle engine. In particular, some parameters were calculated and compared with experimental data available for impulse turbine. They were available test data sufficient to assess of this analytical method accuracy 


\section{Expander Cycle Rocket Engine}

In expander cycle rocket engines, the fuel, once pressurized by the pump, goes through the cooling system that surrounds the combustion chamber and nozzle, where it vaporizes, then flows through the turbine that is used to drive the pump.

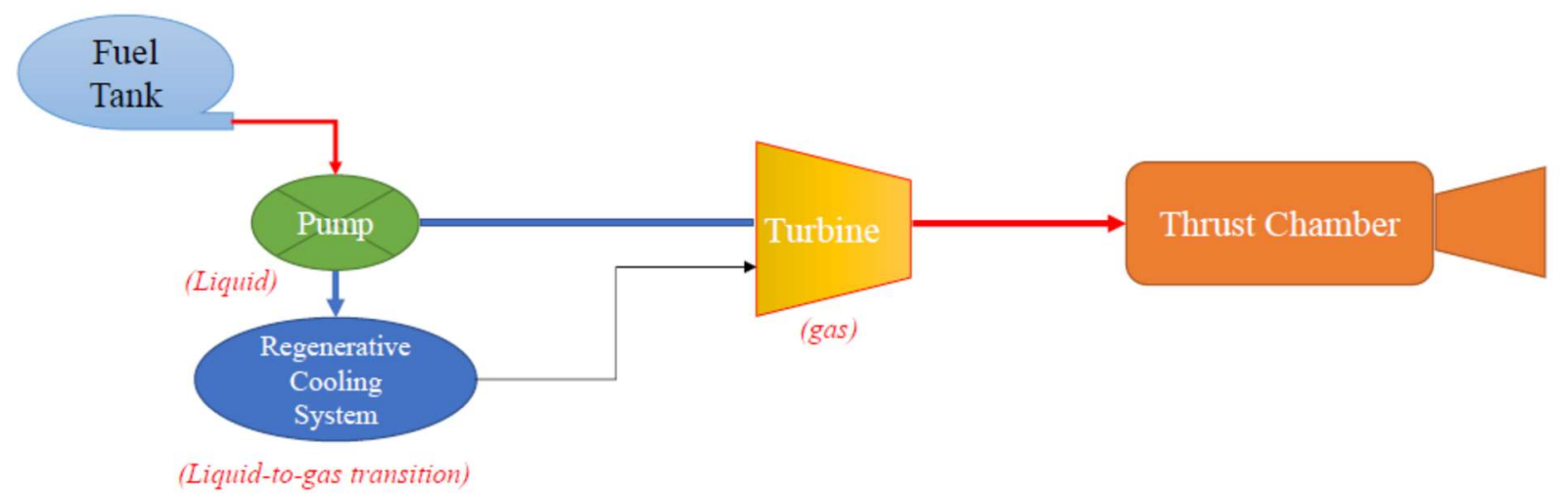

Fig. 1. Expander cycle configuration

In the expander cycle rocket engines are possible the following configurations of turbopump feed system. The power required from the fuel pump and LOX pump is generated by the expansion through the turbine of the vaporized fuel at the exit of the cooling system. The turbopump feed system has only a turbine connected directly to the faster pump (i.e. fuel pump), while it is necessary a speed reduction gear for slower pump (i.e. LOX pump). Typically, the pump of liquid oxygen is slower than that of the fuel, because oxygen has a high density. For instance, this configuration is adopted by RL10A-3-3A engine [2].

Parallel Turbopump: in this case there are two feed systems (two turbopumps), the vaporized fuel is diverted and sent, with different percentages, to two turbines. These turbines are connected directly to two pumps, which will have different rotation speeds, and then through the two turbines will expand a different flow rate of fuel. For instance, this configuration is adopted by VINCI engine [4].

\subsection{RL10-3-3A Engine}

The RL10A rocket engine is an important component of the United States space infrastructure [2]. Two RL10 engines form the main propulsion system for the Centaur upper stage vehicle, which boosts commercial, scientific, and military payloads into Earth orbit and beyond (i.e. planetary missions). This upper stage is used on both Atlas and Titan launch vehicles. In particular, the RL10A-3-3A engine design is based on an expander cycle. Engine schematic is shown in Figure 2. Indeed, the Hydrogen fuel is used to cool the thrust chamber and nozzle, and the thermal energy transferred to the coolant is used to drive the turbo-pumps. The fuel pump consists of two stages; while the LOX pump features an inducer and a single centrifugal impeller, followed by a vaneless diffuser and conical exit volute.

Engine data available in literature for the RL10A-3-3A [2] are summarized in Table 1.

Table 1. Turbine Data RL10A-3-3A

\begin{tabular}{|l|c|}
\hline \multicolumn{1}{|c|}{ Parameter } & Data \\
\hline Number Resolution per Minute [RPM] & 31494 \\
\hline Mass Flow Rate [kg/s] & 2.7469 \\
\hline Turbine Torque [Nm] & 178.27 \\
\hline Turbine Pressure Ratio & 1.402 \\
\hline Total-to-Static Velocity Ratio & 0.4581 \\
\hline Turbine Total Inlet Pressure [bar] & 56.11 \\
\hline Turbine Static Exit Pressure [bar] & 40 \\
\hline Turbine Total Inlet Temperature [bar] & 213.44 \\
\hline Turbine Static Exit Temperature [K] & 191.9 \\
\hline Turbine Enthalpy Drop Dh [kJ/kg] & 295.6 \\
\hline Turbine Efficiency & 0.7353 \\
\hline Turbine Specific Work [kJ/hg] & 214.239 \\
\hline Turbine Power [kW] & 588.515 \\
\hline Total Power Fuel and Oxidizer Centrifugal Pumps Required [kW] & 577.199 \\
\hline
\end{tabular}




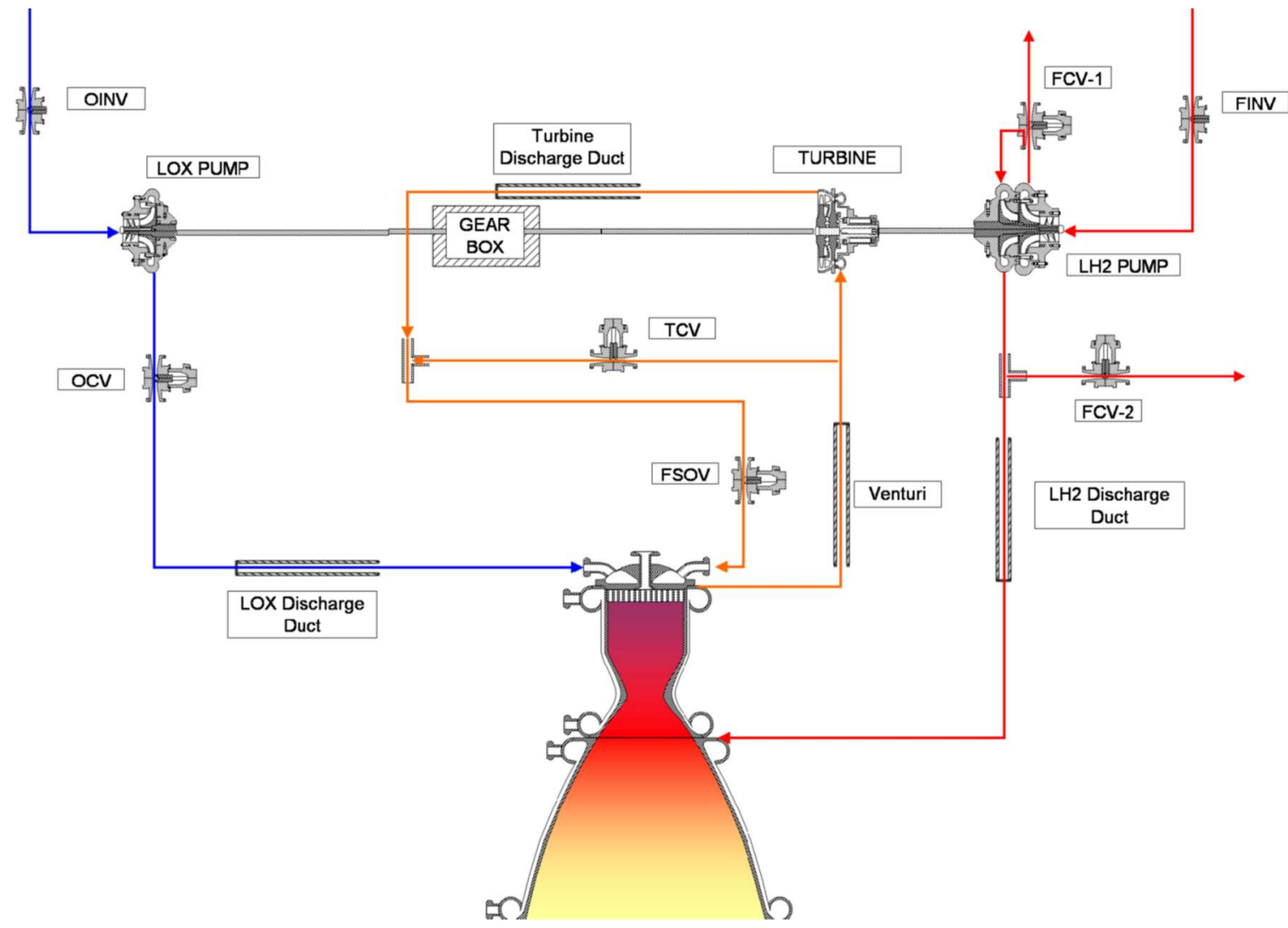

Fig. 2. RL10A-3-3A Engine Diagram

\section{Code Structure}

\subsection{Meanline Method Design}

A simplified procedure for the rotor design consists of the meanline analysing method. This method allows to obtain the main geometric radial turbine parameters, as it requires a small input amount. In addition, it is possible to perform a loss analysis to proceed with the rotor three-dimensional design.

A series of data must be provided to RTGD tool, to start the meanline design calculation. The main input parameters are:

- Turbine inlet total temperature,

- Turbine inlet total pressure,

- Mass flow rate,

- Number of revolutions per minute,

- Rotor inlet flow angle

- Blade material density,

- Material yield strength,

- Young's modulus,

- Poisson's ratio.

From the acquired data combination, listed above, RTGD proceeds with velocity triangles computation, total and static pressure and temperature, inlet and outlet rotor diameter and blade height.

Blades number is obtained considering the Glassman [5] equation:

$$
z=\frac{\pi}{30}\left(110-\alpha_{1}\right) \tan \alpha_{1}
$$

The previous equation depends on the $\alpha_{1}$ absolute flow angle, which is generally designer imposed.

The inlet and outlet blade thickness are defined according to the trailing edge thickness. They represent respectively $4 \%$ and $2 \%$ of the rotor input radius [6].

A similar approach has been used to calculate axial and radial clearance, assuming a range between $5 \%$ and $10 \%$ of respective shroud blade height. The radial clearance was found to make a larger contribution to the secondary flow phenomena generation and exit flow angle deviation than the axial clearance. The axial and radial clearance are necessary for tip clearance loss estimation [7]. 
The components of rotor inlet velocity triangle are related by the following equation that depends on loading coefficient:

$$
\psi^{2}-2 \psi \sin ^{2} \alpha_{1}+\sin ^{2} \alpha_{1}\left(1-\left(\frac{w_{1}}{u_{1}}\right)^{2}\right)=0
$$

The correct value of $\psi$ can be verified if the following condition is respected:

$$
\cos ^{2} \alpha_{1} \leq\left(\frac{w_{1}}{u_{1}}\right)^{2}
$$

Therefore, RTGD will estimate the loading coefficient value from the equation:

$$
\psi=\sin ^{2} \alpha_{1} \pm \sin ^{2} \alpha_{1} \sqrt{\left(\frac{w_{1}}{u_{1}}\right)^{2}-\cos ^{2} \alpha_{1}}
$$

If $\frac{w_{1}}{u_{1}}=\cos \alpha_{1}$ the maximum $\psi$ value is:

$$
\psi=\sin ^{2} \alpha_{1}
$$

The flow coefficient will be calculated as a $\psi$ function

$$
\varphi=\frac{\psi}{\tan \alpha_{1}}
$$

The maximum efficiency occurs when the loading coefficient is in the range 0.9-1.0 and the flow coefficient is between about $0.2-0.3$. However, in many cases, designers must use significantly different values than optimum, mainly because the application limits the design. For example, turbopumps must use a higher flow coefficient to reduce the outlet blade height to achieve a more compact design. Therefore, it becomes difficult to quickly determine the maximum efficiency that the project can achieve with specific constraints.

In this case, given as input the rotor inlet flow angle, the flow and loading coefficients are determined by RTGD tool which generates a diagram that links the efficiency, the flow and loading coefficients to the rotor inlet flow angle.

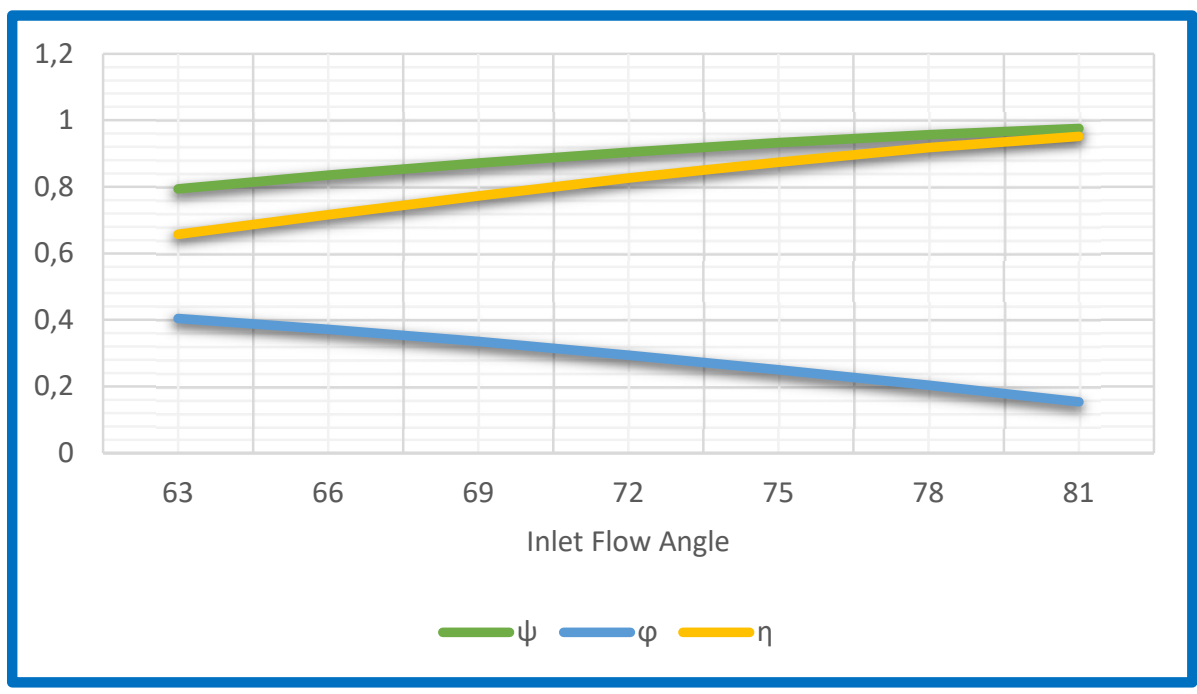

Fig. 3. Inlet Flow Angle Diagram [5]:

Finally, RTGD will determine the mean surface length as a chord function through the following geometric relationships

$$
c=\frac{1.5 h_{2}}{\cos \beta_{a v}}
$$

where: 


$$
\tan \beta_{a v}=\frac{\tan \beta_{1}-\tan \beta_{2}}{2}
$$

Finally:

$$
L_{m s}=\frac{c \pi}{2 \sqrt{2}}
$$

Since static conditions are invariant for relative and absolute flow the relative Mach number, $M_{2}$, is also known. The mean blade pitch at the rotor exit is given by:

$$
q_{2}=\frac{2 \pi r_{s 2}}{z}
$$

If $M_{2} \leq 1$, the rotor mean throat width will be:

$$
o_{2}=\frac{q_{2} c_{2}}{w_{2}}
$$

These parameters are necessary to estimate the passage loss [7].

\subsection{Performance Prediction}

In this section will be presented the total-to-static efficiency as a function of the total enthalpy drops and losses:

$$
\eta_{\mathrm{ts}}=\frac{\Delta \mathrm{h}_{0}-\sum \Delta \mathrm{h}_{\text {loss }}}{\Delta \mathrm{h}_{0}}
$$

This efficiency value must converge with that on the diagram, Figure 3, with an error margin of $\pm 5 \%$.

RTGD will investigate different types of losses: incidence, passage, tip-clearance, trailing edge, windage losses and kinetic-energy loss at the rotor exit.

Most internal rotor losses are fluid velocity function and to improve the rotor efficiency the velocity at the rotor inlet should be reduced as much as possible to reduce the incidence loss that occurs at the rotor inlet. The loading coefficient would be used to minimize the fluid velocity at the rotor inlet.

The incidence losses refer to the losses that occur at the inlet of the radial inflow turbine rotor blade passages when the turbine is operating at a non-zero incidence and, therefore, the flow does not enter the passage in the optimum direction. Losses in the rotor blade passage are mainly due to the occurrence of secondary flows, and these are considerably affected by the flow deviation from optimum incidence.

The minimum loss for incidence can be determined as a function of the relative tangential velocity:

$$
\Delta \mathrm{h}_{\mathrm{i}}=\frac{1}{2} \mathrm{w}_{\mathrm{u} 1}^{2}
$$

The incidence angle is obtained from the difference between the inlet flow angle and inlet optimum flow angle $\beta_{1 \_ \text {opt }}$ [5]. The optimal relative flow angle of the rotor inlet must be included as reported by Baines [7] between $-20^{\circ}$ and $-40^{\circ}$. According Reference [8] the incidence angle value calculated is constant $-32^{\circ}$ in the case examined in this work

The term passage losses include a wide spectrum of different phenomena occurring to the fluid crossing the rotor. In fact, after a rapid acceleration in the flow direction, the fluid is turned in the meridional plane along the camber line: this creates a complex pattern of secondary and cross-stream flows, which still today are not completely understood. Moreover, this causes the growth of boundary layers with loss of kinetic energy and blockage. A fully detailed model that considers separately all these loss sources, as the ones existing for the axial turbines, has not yet been developed. In fact, in axial turbine cascades, this can be done by a careful set up and measures, but this is not actually possible for radial turbines, due to the three-dimensionality of the flow pattern, which does not permit to differentiate the losses.

A passage loss model, namely the CETI model [8], was implemented to estimate more realistically the losses due to the secondary flow and friction in the rotor passages, between the inlet and the exit throat section of the rotor.

In this model, secondary flow and friction loss formulations are combined into one correlation as:

$$
\Delta h_{p}=0.11 \frac{w_{1}^{2}+w_{t}^{2}}{2}\left(\frac{L_{h}}{D_{h}}+0.68\left(1-\left(\frac{r_{t}}{r_{1}}\right)^{2}\right) \frac{\cos \left[\beta_{t}\right]}{\frac{h_{t}}{c}}\right)
$$

where $L_{h}$ and $D_{h}$ represents hydraulic length and hydraulic diameter respectively, the subscript $t$ denotes the throat. 
The impeller blades mate up against the turbine housing with a small clearance to avoid mutual contact. In addition, the fluid pushes on the leading surface of each blade essentially creating a pressure difference between the leading and trailing blade surfaces i.e. across the blade. This pressure difference gives rise to a flow through the blade-housing clearance gap. This flow results in pressure dissipation and a consequent loss.

In this paper the clearance loss is calculated using Baines [7] equation, model that consider the axial and radial clearances influences:

$$
\Delta \mathrm{h}_{\mathrm{c}}=\frac{\mathrm{u}_{1}^{3} \mathrm{z}}{8 \pi}\left(\mathrm{K}_{\mathrm{a}} \varepsilon_{\mathrm{a}} \frac{1-\frac{\mathrm{r}_{2}}{\mathrm{r}_{1}}}{\mathrm{c}_{\mathrm{r} 1} \mathrm{~b}_{1}}+\mathrm{K}_{\mathrm{r}} \varepsilon_{\mathrm{r}} \frac{\mathrm{r}_{2}}{\mathrm{r}_{1}} \frac{\mathrm{z}_{\mathrm{a}}-\mathrm{b}_{1}}{\mathrm{c}_{2} \mathrm{r}_{2} \mathrm{~b}_{2}}\right)
$$

where $\mathrm{z}_{\mathrm{a}}$ is the axial length, $\varepsilon_{\mathrm{a}}$ and $\varepsilon_{\mathrm{r}}$ represent axial and radial clearance, $\mathrm{K}_{\mathrm{a}}$ and $\mathrm{K}_{\mathrm{r}}$ are discharge coefficients for the axial and radial tip clearances respectively.

Trailing edge losses [9] arises due to mixing occurring, as the adjacent rows, of the two-blade surfaces exit the blade row. Moreover, in the case at supersonic velocities shock losses also contribute. In the fourth step the RTGD code determine the trailing edge loss as a function of the exit Mach number, the exit pressure $\mathrm{p}_{2}$ and temperature $\mathrm{T}_{2}$, the relative velocity rotor exit $\mathrm{w}_{2}$ and relative pressure loss $\Delta \mathrm{p}_{0}$, as follows:

$$
\Delta \mathrm{h}_{\mathrm{t}}=\frac{2}{\gamma \mathrm{M}_{2}^{2}} \frac{\Delta \mathrm{p}_{0}}{\mathrm{p}_{2}\left(1+\frac{\mathrm{w}_{2}^{2}}{2 \mathrm{~T}_{2} \mathrm{C}_{\mathrm{p}}}\right)^{\frac{\gamma}{\gamma-1}}}
$$

Windage losses [9] are frictional losses occurring on the back face of the turbine disk:

$$
\Delta \mathrm{h}_{\mathrm{w}}=\mathrm{K}_{\mathrm{f}} \frac{\rho_{\mathrm{avg}} \mathrm{u}_{2}\left(\frac{\mathrm{D}_{2}}{2}\right)^{2}}{\mathrm{~g} \mathrm{~m} \mathrm{w}_{2}^{2}}
$$

where the friction coefficient $\mathrm{K}_{\mathrm{f}}$ is a function of Reynolds number.

The only external loss that is usually considered in radial turbine modelling is that of disk friction. This occurs because of the fluid leakage between the rotor disc and the stationary back plate, where the windage flow causes quite strong friction. Depending on the turbine, this leakage could also be recirculated into the turbine annulus or taken away.

The back face of the impeller hub is an annular disk that spins with the impeller. Either this disc may mate with a fixed surface separated from it by a small clearance or it may spin freely far from other fixed surfaces. In either case, the fluid adjacent to the disc exerts a shear on the disc with result that the fluid does unproductive work.

Finally, the rotor exit kinetic loss is expressed as:

$$
\Delta \mathrm{h}_{\mathrm{k}}=\frac{1}{2} \mathrm{c}_{2}^{2}
$$

Figure 4 shows the losses contribution for RL10-3-3A engine.

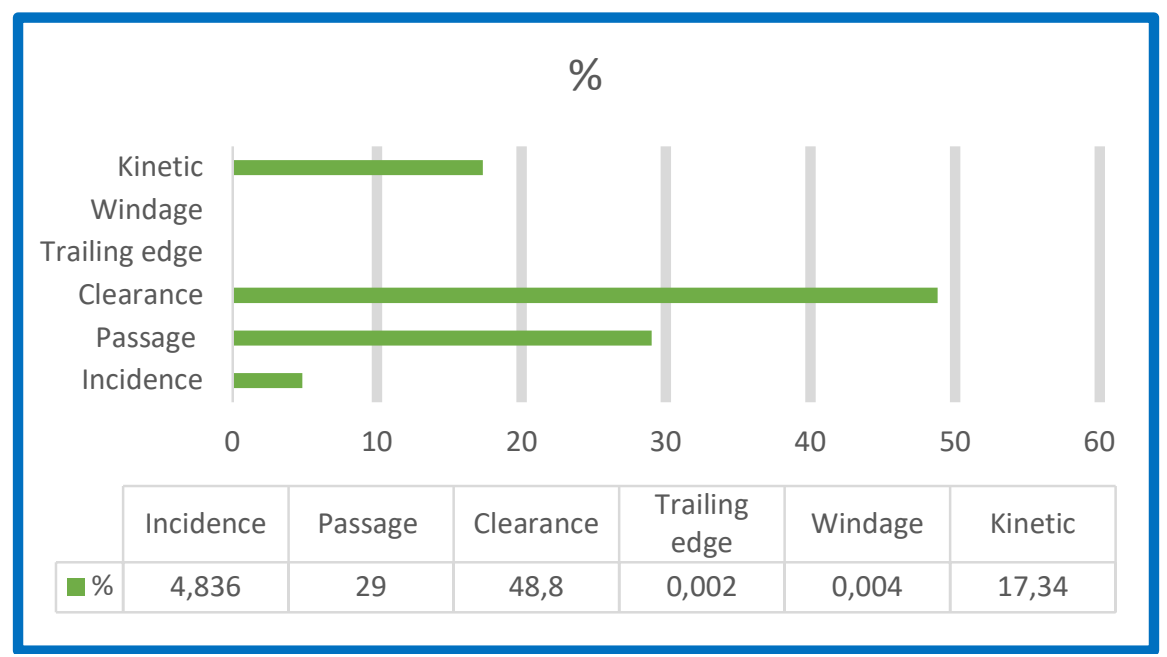

Fig. 4. RL10-3-3A Loss Models

\subsection{Geometry Rotor Design}

In the RTGD model used in this paper, geometric constraints are implemented to ensure a reliable rotor geometric design. 
The minimum requirements for the rotor geometry to be considered feasible, the ratio between shroud and inlet diameters must be less than 0.78 [10]. To avoid too large curvatures of impeller flow lines near the casing, Rohlik suggest that $\mathrm{D}_{\mathrm{s} 2}$ is at most equal to $0.7 \mathrm{D}_{1}$ [11].

Another constraint is represented by the relationship between hub and shroud diameters, which according to Rohlik [11] must be $\geq 0.4$, in order to limit both the excessive blades blockage on the obstruction factor and the losses that occur near the hub. Another geometric constraint is the ratio between average rotor exit diameter and inlet diameter whose range is $0.2 \div 0.6[11]$. Finally, the ratio between the absolute exit velocity and the tangential inlet velocity must be between 0.2 and $0.4[10]$.

These ratios are represented in the Table 2 as a comparison with the RTGD tool results.

Table 2. Ratio Maximum Performance

\begin{tabular}{|c|c|c|c|}
\hline Ratio & Rohlik & Balje & RTGD \\
\hline$\frac{D_{s 2}}{D_{1}}$ & $\leq 0.7$ & $\leq 0.78$ & 0.32 \\
\hline$\frac{D_{h 2}}{D_{s 2}}$ & $\geq 0.4$ & & 0.62 \\
\hline$\frac{D_{2}}{D_{1}}$ & $0.2 \div 0.6$ & & 0.26 \\
\hline$\frac{c_{2}}{u_{1}}$ & & $0.2 \div 0.4$ & 0.25 \\
\hline
\end{tabular}

If the rotor geometric results respect these constraints, the RTGD tool will continue with the subsequent calculations.

In addition, RTGD considers rotor blades elastic stress [12] due to centrifugal loading, which must be lower by a safety factor than the allowable tension of material chosen for the blades manufacture:

$$
\sigma_{r}=K_{g} \rho_{m} u_{1}^{2}
$$

where $\rho_{\mathrm{m}}$ is the material density and $\mathrm{K}_{\mathrm{g}}$ is a geometric factor equal 0.3 .

Another rule based on experience concerns the rotor frequency, which has to be at least four times higher than the first bending mode [13]:

$$
\omega_{n}=\frac{6.94}{2 \pi h_{2}^{2}} \sqrt{\frac{E t^{3}}{12 \rho_{m}\left(1-v^{2}\right)}}
$$

\section{Results Discussion}

In this section, the results obtained with RTGD tool will be presented and compared with the RL10-3-3A engine literature data.

The RTGD tool will determined in the first step imposed $\alpha_{1}$, the nominal efficiency as a function of loading coefficient.

In the second step, the inlet and outlet velocity triangles will be computed according to the flow and loading coefficients.

In the third step, the rotor geometry will be estimated. In the fourth step, the six losses types will be calculated, once the losses are obtained, efficiency will be predicted.

Finally, RTGD performs a static structural analysis due to the high rotational speed. For the radial turbine design, the Inconel 718 alloy was chosen for its high mechanical properties.

The yield strength is defined as the stress at which a material starts to deform plastically. Inconel 718 has a yield strength of 1172 [MPa]. A safety factor of 1.4 was considered that reduces the yield strength.

The design results and comparison are provided in Table 3.

Table 3. RTGD design results and comparison with the NASA results for RL10-3-3A engine

\begin{tabular}{|l|c|c|}
\hline \multicolumn{1}{|c|}{ Parameter } & RTGD & RL10 Axial Turbine \\
\hline$\eta_{\text {ts }}$ & 0.81 & 0.7333 \\
\hline Pressure Ratio & 1.33 & 1.402 \\
\hline Specific Work $[\mathrm{kJ} / \mathrm{kg}]$ & 212.13 & 214.239 \\
\hline$\Delta \mathrm{h}[\mathrm{kJ}]$ & 251.64 & 295.6 \\
\hline Power $[\mathrm{kW}]$ & 582.95 & 588.515 \\
\hline Exit Pressure $[\mathrm{bar}]$ & 42.18 & 40 \\
\hline \multicolumn{2}{|r|}{} \\
\hline
\end{tabular}


The results show that the radial turbine is more advantageous compared to the axial one. In particular, the radial turbine turns out to be advantageous since it has a higher efficiency and a lower expansion ratio than the axial turbine. Both priority requirements for a turbomachinery expander cycle rocket engine.

Table 4 shows the design results obtained from the meanline design process.

Table 4. Design Results

\begin{tabular}{|l|c|}
\hline \multicolumn{1}{|c|}{ Parameter } & Design Results \\
\hline Rotor Total Inlet Temperature $\mathrm{T}_{11}[\mathrm{~K}]$ & 212.7 \\
\hline Rotor Static Inlet Temperature $\mathrm{T}_{1}[\mathrm{~K}]$ & 203.7 \\
\hline Rotor Total Exit Temperature $\mathrm{T}_{02}[\mathrm{~K}]$ & 212.06 \\
\hline Rotor Static Exit Temperature $\mathrm{T}_{2}[\mathrm{~K}]$ & 195.9 \\
\hline Rotor Total Inlet Pressure $\mathrm{p}_{01}[\mathrm{bar}]$ & 55.7 \\
\hline Rotor Static Inlet Pressure $\mathrm{p}_{1}[\mathrm{bar}]$ & 48.4 \\
\hline Rotor Total Exit Pressure $\mathrm{p}_{02}[\mathrm{bar}]$ & 55.15 \\
\hline Rotor Static Exit Pressure $\mathrm{p}_{2}[\mathrm{bar}]$ & 42.18 \\
\hline Rotor Inlet Radius $\mathrm{r}_{1}[\mathrm{~m}]$ & 0.148 \\
\hline Rotor Shroud Radius $\mathrm{r}_{2}[\mathrm{~m}]$ & 0.048 \\
\hline Rotor Exit Hub Radius $\mathrm{r}_{2}[\mathrm{~m}]$ & 0.029 \\
\hline Rotor Axial Length $\mathrm{Z}_{\mathrm{r}}[\mathrm{m}]$ & 0.027 \\
\hline Rotor Inlet Height $\mathrm{h}_{1}[\mathrm{~m}]$ & 0.0038 \\
\hline Rotor tip velocity at rotor inlet $\mathrm{u}_{1}[\mathrm{~m} / \mathrm{s}]$ & 491.55 \\
\hline Flow relative velocity at rotor inlet $\mathrm{w}_{1}[\mathrm{~m} / \mathrm{s}]$ & 150.1 \\
\hline Absolut flow velocity at rotor inlet $\mathrm{c}_{1}[\mathrm{~m} / \mathrm{s}]$ & 467.58 \\
\hline Rotor tip velocity at rotor exit $\mathrm{u}_{2}[\mathrm{~m} / \mathrm{s}]$ & 158.64 \\
\hline Flow relative velocity at rotor exit $\mathrm{w}_{2}[\mathrm{~m} / \mathrm{s}]$ & 177.6 \\
\hline Absolut flow velocity at rotor exit $\mathrm{c}_{2}[\mathrm{~m} / \mathrm{s}]$ & 122.76 \\
\hline Specific Speed $\mathrm{N}_{\mathrm{s}}$ & 0.22 \\
\hline Relative flow angel at rotor inlet $\beta_{1}\left[{ }^{\circ}\right]$ & 23 \\
\hline Number Blades & 12 \\
\hline Incidence Angle $\left[{ }^{\circ}\right]$ & -32 \\
\hline Chord c [m] & 0.032 \\
\hline Mean Surface Length $\mathrm{L}_{\mathrm{ms}}[\mathrm{m}]$ & 0.092 \\
\hline Rotor Blades Elastic Stress $\sigma_{\mathrm{r}}\left[\mathrm{N} / \mathrm{m}^{2}{ }^{2}\right]$ & $593 \cdot 10^{6}$ \\
\hline Yield Strength with Safety Factor $[\mathrm{MPa}]$ & $885.7 \cdot 10^{6}$ \\
\hline Frequency [Hz] & 524.9 \\
\hline Mach M & 0.16 \\
\hline
\end{tabular}

\section{Conclusions}

In this paper, radial turbine was examined in order to make a comparison in terms of performance with the axial turbine used in the RL10 engine. Therefore, an automated preliminary design procedure for radial flow turbines was presented as the integrated meanline analysis and optimization approach using the RTGD tool.

RTGD was developed in order to release the radial turbine design from the designer's experience dependence. The purpose of this design approach is to obtain the best design with high aerodynamic performance as a function of the loading and flow coefficients, and rotor inlet flow angle.

Several loss models have been incorporated into the code, which showed that the passage, tip clearance and kinetic losses account for most of the overall losses compared to the other loss mechanisms such as: incidence, trailing edge and windage.

In addition to a losses analysis a static structural analysis has been implemented in order to verify the chosen material strength.

It has been demonstrated that it is indeed possible to use single-stage radial turbines to drive the turbo-pumps that supply hydrogen and oxygen to the thrust chamber of a rocket engine with characteristics similar to the RL10 engine.

The radial turbine design obtained with RTGD showed a high efficiency and a lower expansion ratio, priority requirements for a turbomachinery expander cycle rocket engine. In particular, it has been found an improvement in both the efficiency and the expansion ratio about $10 \%$.

A possible future development will be the $3 \mathrm{D}$ rotor geometric generation by RTGD and a computational fluid dynamics analysis. 


\section{References}

1. A. Leto, Radial Turbine Global Design for Liquid Rocket Engine Application (ETC2019-324, 2019)

2. M. Binder, RL10A-3-3A Rocket Engine Modelling Project (NASA Technical Memorandum 107318, 1997)

3. https://pubs.acs.org/doi/abs/10.1021/ie4033999

4. www.ariane.group

5. A. J. Glassman, Computer Program for Design Analysis of Radial- Inflow Turbines (NASA, Technical Note No. TN D-8164, 1986)

6. R. H. Aungier, Turbine Aerodynamics, Axial-Flow and Radial-Inflow Turbine Design and Analysis (ASME Press, New York, 2006)

7. H. Moustapha, M. F. Zelesky, N. C. Baines D. and Japikse, Axial and Radial Turbines (Concepts NREC, White River Junction, 2003)

8. N. C. Baines, Low Development in Radial Turbine Rotors (The American Society of Mechanical Engineers, 1996)

9. S.K. Ghosh, R. K. Sahoo and S. K. Sarangi, Mathematical Analysis for Off-Design Performance of Cryogenic Turboexpander Trans (ASME Journal of Fluids, 2011)

10. O. E. Balje, Turbo a Guide to Design, Selection and Theory (Winley, 1981)

11. H. E. Rohlik, Analytical determination of radial inflow turbine design geometry for maximum efficiency (NASA TN D-4384, 1968)

12. W. D. Marscher, 1992, Structural Analysis: Stresses due to Centrifugal, Pressure and Thermal Loads in Radial Turbines, (von Karman Institute for Fluid Dynamics VKI Lecture Series, Radial Turbines, SEE N93-10050 01-34, 1992)

13. R. B. Blevins, Formulas for Natural Frequency and Mode Shape (van Nostrand Reinhold Company, New York, 1979) 